\title{
PROBABILISTIC MODELING OF ABOVEGROUND STORAGE TANKS UNDER SURGE AND WAVE LOADS
}

\author{
Carl Bernier ${ }^{1}$ and Jamie Padgett ${ }^{1}$
}

\begin{abstract}
This study presents the development of probabilistic models to assess the structural performance of a typical aboveground storage tank (AST) subjected to storm surge and wave loads. First, a finite element model is developed and validated against experimental results to determine hydrodynamic loads on the AST. This finite element model is then employed to derive a regression model of the hydrodynamic loads across ranges of surge and wave parameters using an Artificial Neural Network. This regression model is used as a surrogate of the finite element model to facilitate the investigation of the structural behavior of the case study AST. Finally, the buckling behavior of the AST and the stability of the tank to dislocation (uplift, overturning, or siding) are assessed for various AST modeling parameters and load conditions in order to develop fragility models. Two distinct fragility models are derived, one for dislocation and one for buckling. Key insights on the influence of surge and wave loads are obtained from these models. Results indicate that wave loads and hydrodynamic effects are significant, and neglecting them could underestimate the probability of dislocation or buckling of the AST by up to $30 \%$. Overall, this paper proposes a rigorous yet efficient methodology for the fragility modeling of ASTs during storm events and opens the path for future investigations of the performance of ASTs with a range of design details and exposure conditions.
\end{abstract}

Keywords: storm surge; wave loads; aboveground storage tank; buckling; flotation; fragility model

\section{INTRODUCTION}

Aboveground storage tanks (ASTs) are a key component of refineries, ports, and industrial facilities as they allow the storage of raw and refined products. ASTs are generally constructed from welded thin steel plates forming a vertical cylinder and can easily hold millions of liters of hazardous chemicals. Their thin-walled cylindrical geometry makes them lightweight, economical, and able to efficiently withstand pressure from internal liquids (Myers 1997). However, this geometric slenderness and light weight also make them vulnerable to external loads such as wind, flooding and storm surge. This vulnerability was exposed during Hurricanes Katrina and Rita, during which approximately 20 ASTs buckled or floated away under surge, flood, and wind effects, releasing more than 26 million liters of oil into the surrounding environment (Godoy 2007, Santella et al. 2010). To a lesser extent, the potential for AST failures was also observed during Hurricanes Ike, Gustav, and Isaac (Hyder 2008, Pardue 2013). More recently, during Hurricane Harvey, approximately two million liters of crude oil, gasoline, or other petrochemicals were spilled due to the failure of ASTs (Bernier and Padgett 2018a). Such ASTs failures generally result in serious environmental damage to wildlife and sensitive ecosystems due to the spillage of hazardous materials, and economic impacts due to clean-up costs, lawsuits, disruption of port activities, and production losses. In addition, AST failures can have cascading effects on the mental and physical wellbeing of nearby communities (Palinkas et al. 1993).

Post-storm investigations usually report three main failure modes for ASTs: (i) wind-induced buckling (Godoy 2007, Godoy 2016); (ii) surge- or flood-induced buckling (Cozzani et al. 2010); and (iii) dislocation of the AST from the ground due to storm surge or flood effects (Godoy 2007, Cozzani et al. 2010). However, in past decades, most of the existing literature assessing the structural performance of ASTs during storm events has mainly focused on wind-induced buckling, with very few studies looking at the performance of ASTs under storm surge loads. Moreover, the few studies that consider surge loads (i.e., Landucci et al. 2012, Kameshwar and Padgett 2018) only considered the hydrostatic effects of storm surge and neglected potentially significant hydrodynamic loads such as current and waves. At the exception of a few studies on tsunami (Sakakiyama 2009), the behavior of ASTs under such hydrodynamic loads has received little attention to date.

Thus, the objective of this paper is to investigate the structural behavior and to develop probabilistic models of the performance of a typical AST under hydrostatic and hydrodynamic loads during storm surge. The typical AST considered reflects the median geometry of ASTs located in the Houston Ship Channel (HSC), the largest petrochemical complex located in a hurricane prone region in the US. The case study AST has a diameter of $15 \mathrm{~m}$ and a height of $10 \mathrm{~m}$. First, this paper presents the development of a three-dimensional finite element (FE) model that takes into account fluid-structure interaction (FSI) to compute hydrodynamic loads acting on the AST. The validation of the finite 
element model against experimental results is also presented. Then, based on a series of finite element analyses, a surrogate model is derived to facilitate the estimation of hydrodynamic loads on the AST across ranges of surge and wave conditions. The ranges of surge and wave conditions are obtained from numerical simulations of historic and synthetic hurricanes in and around the Houston, Texas region in the US. Next, with this surrogate model, the structural behavior of the AST during storm surge is investigated for various AST modeling parameters and load conditions. Finally, fragility models, which indicate the conditional probability of failure of a structure given a hazard level and set of structural characteristics, are developed. Fragility models are developed for the two failure modes related to storm surge and wave loads: dislocation of the AST from the ground and buckling of the tank shell. The effects of hydrodynamic loads on the structural performance of the AST are also discussed, providing useful insights for future probabilistic studies of AST performance during storm events.

\section{NUMERICAL MODELING OF HYDRODYNAMIC LOADS ON THE AST}

In order to determine the structural behavior of an AST subjected to surge and wave loads and to develop fragility models, an adequate estimation of the hydrodynamic pressure acting on it is first required. Currently, there is little information available regarding hydrodynamic loads, such as current and waves, on ASTs. Existing hydrodynamic load models for vertical cylindrical structures, such as the Morison equation (Morison et al. 1950) or diffraction theory (Kriebel 1990), were mainly developed for small-scale cylinders, deep sea conditions, or low-order wave theories. However, ASTs are large-scale cylinders that could be subjected to high-order and shallow water waves during storm surge events. Thus, to overcome the lack of adequate load models for large cylindrical structures such as ASTs, this study relies on the finite element (FE) method to estimate the hydrodynamic loads acting on the case study AST.

The FE model employed here to estimate hydrodynamic loads on ASTs is presented in Fig. 1 and was developed using the Arbitrary Lagrangian-Eulerian (ALE) method in LS-Dyna (LSTC 2015). In this method, water and air fluids are governed by the Navier-Stokes equations while an ALE formulation tracks the water-air interface. Water is modeled using a fluid-like material with the physical properties of water, while air is modeled as a vacuum-like material to reduce the computational cost. The interface between the fluids and the tank shell is modeled with a multi-physics contact allowing fluid-structure interactions (FSI). For assessing the hydrodynamic loads, the tank shell is assumed to be rigid, and only half of the domain is modeled. While these assumptions greatly reduce the computational cost of the model, they do not significantly affect its accuracy; differences in the pressure distributions of less than $5 \%$ were observed with a full domain and elastic model. Current and waves are generated using a prescribed velocity boundary condition at the inflow boundary and are absorbed at the outflow boundary using non-reflecting ambient elements. Additional details regarding the numerical model can be found in Bernier and Padgett (2018b).

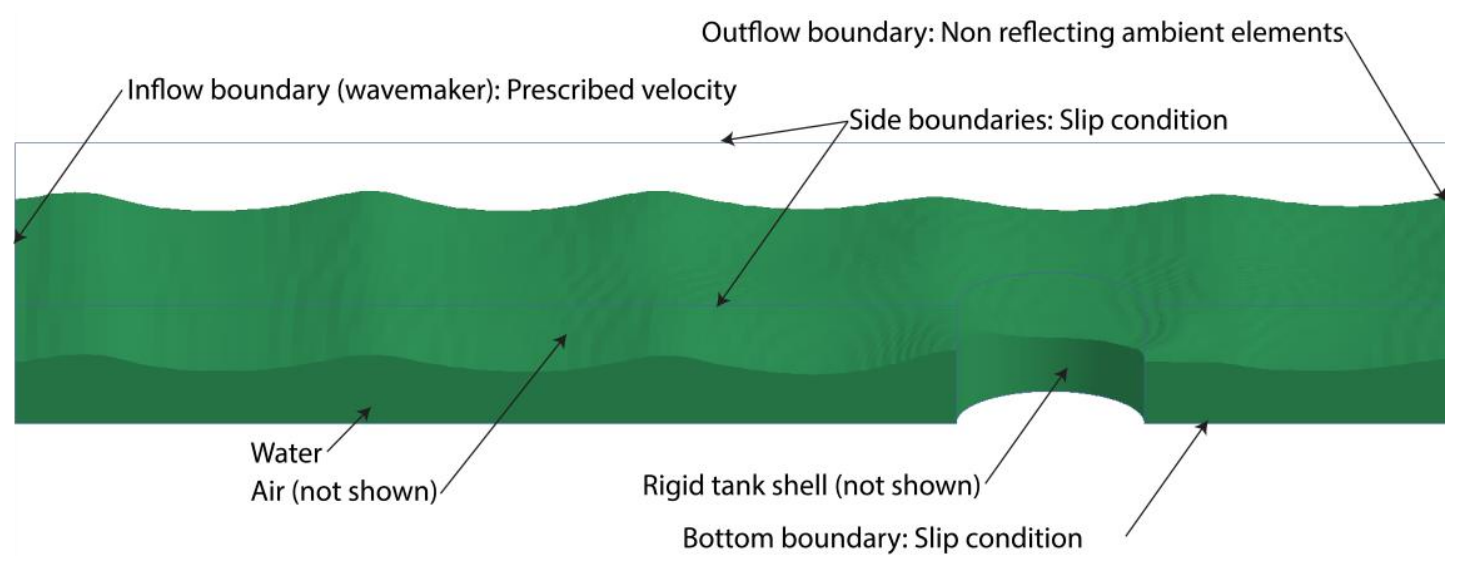

Figure 1. Overview of the finite element model to estimate wave loads on the case study AST.

The velocity profiles used to generate current and waves in the numerical model are obtained from Fenton's Fourier series wave theory (Fenton 1988); this theory is efficient to represent the wide range of wave parameters - wave height $\left(H_{w}\right)$, water depth $(S)$, wavelength $\left(L_{w}\right)$, and wave period $\left(T_{w}\right)-$ considered here as well as to consider the interactions between waves and current $(U)$. For a FE analysis, a total of twenty waves are generated to ensure a steady solution and the hydrodynamic 
pressure distribution is extracted around the AST as detailed further below. To ensure an adequate generation and propagation of the waves, the velocity profiles and the water surface elevations in the numerical model are compared with the theoretical solutions from Fenton's wave theory. Fig. 2 presents this comparison for a wave with $S=5.0 \mathrm{~m}, H_{w}=1.0 \mathrm{~m}$, and $T_{w}=4.0 \mathrm{~s}$.

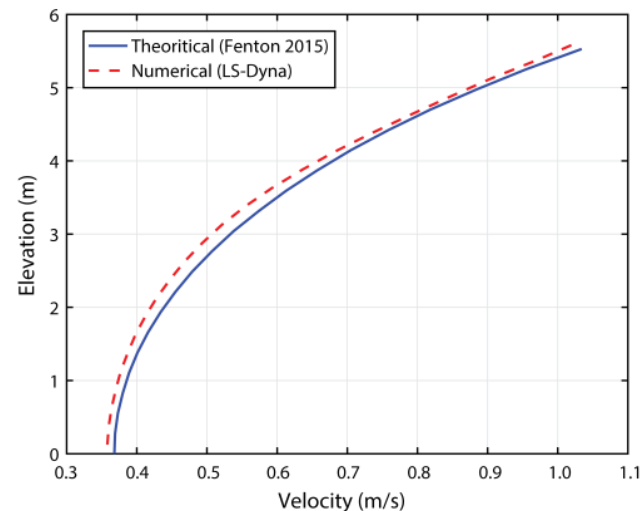

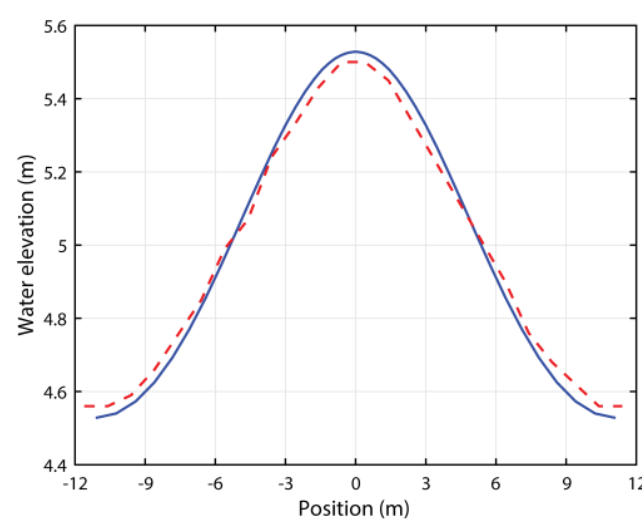

Figure 2. Comparison of: a) horizontal water profile; and b) water surface elevation between the numerical model and theoretical solution for $S=5.0 \mathrm{~m}, H_{w}=1.0 \mathrm{~m}$, and $T_{w}=4.0 \mathrm{~s}$.

The above modeling assumptions are further validated against the experimental results presented in Bernier et al. (2017). Experiments involving a large-scale vertical cylinder subjected to various wave conditions were conducted at the O.H. Hinsdale Wave Research Laboratory at Oregon State University. Sensors were used to measure pressure on the cylinder and water surface elevation and velocity around the cylinder. The experimental conditions were reproduced in LS-Dyna for three solitary wave conditions $\left(H_{w}=0.1,0.2\right.$, and $0.3 \mathrm{~m}$, and $\left.S=0.5 \mathrm{~m}\right)$. Experimental and numerical time histories are compared in Fig. 3 for the cases with $H_{w}=0.1$ and $0.3 \mathrm{~m}$, showing results for the sensors located directly in front of the cylinder. As observed in Fig. 3, the results match very well between the experimental data and the LS-Dyna numerical model. Moreover, the overall results for all other sensor locations and wave conditions indicate differences of less than $5 \%$ for the peak pressure, velocity, and surface elevation. These results indicate that the developed LS-Dyna model can be confidently employed to model waves and estimate the hydrodynamic pressure on the case study AST.

Solitary wave - $0.1 \mathrm{~m}$ :
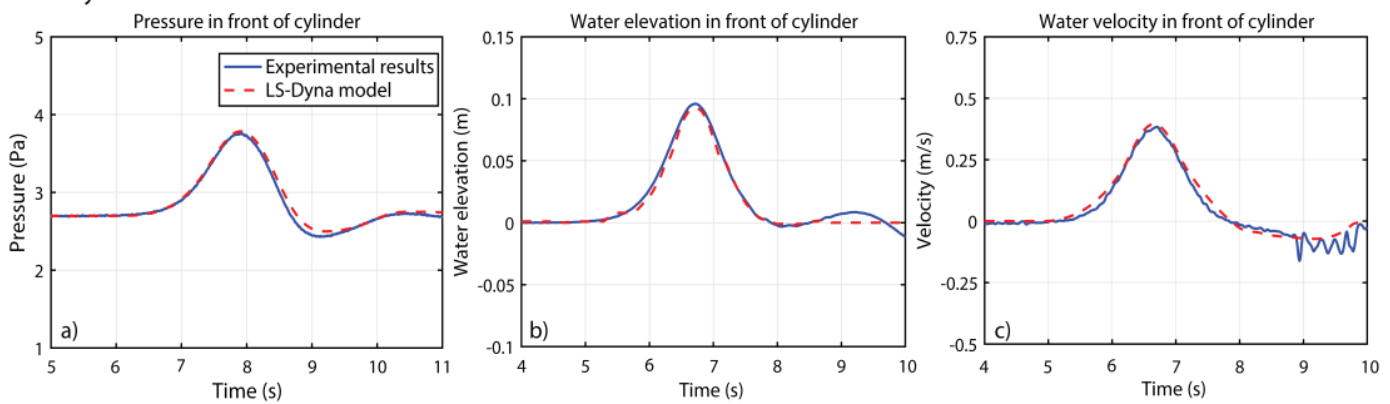

Solitary wave - $0.3 \mathrm{~m}$ :
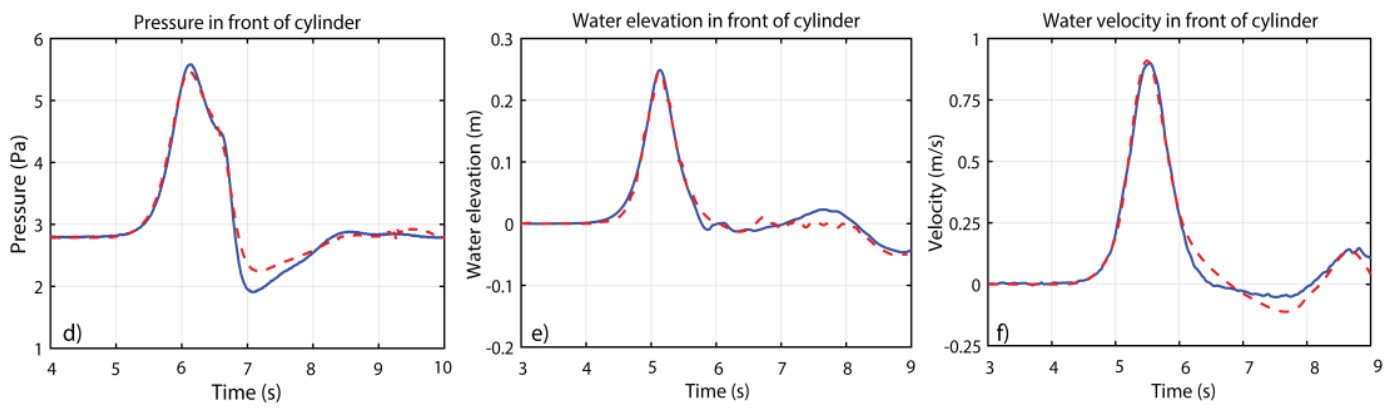

Figure 3. Validation of the numerical model for solitary waves with $H_{w}=0.1$ and $0.3 \mathrm{~m}$. 


\section{SURROGATE MODELING TO ESTIMATE HYDRODYNAMIC LOADS}

Even though the FE model presented in the previous section can provide accurate estimates of hydrodynamic loads, the computational cost associated with this model is a major drawback to investigate the structural behavior of the case study AST and to develop fragility models across ranges of surge and wave parameters. A single analysis with the previous FE model requires approximately 25 CPU-hour, and thousands of analyses will be required to develop the fragility models as detailed in the next sections. Therefore, a regression model is developed based on the output of the FE model to quickly estimate the hydrodynamic loads as a function of the wave parameters (i.e., $S, H_{w}, T_{w}$, and $U$ ). The development of an adequate regression model requires enough training points to efficiently span the space of the wave parameters. To do so, Latin Hypercube Sampling (LHS) is used to generate a space filling experimental design (McKay et al. 1972). The ranges of wave parameters considered in the experimental design are presented in Table 1; these ranges were obtained from numerical simulations of historic and synthetic hurricanes in the Houston area (Dawson 2017). A total of 220 samples with different surge and wave conditions were generated using LHS; 200 samples were used to train the regression model, while 20 were used to test it. When generating the samples, the ratios $H_{w} / S$ and $H_{w} / L_{w}$ were limited to 0.65 and 0.14 respectively to ensure the generation of realistic waves (Fenton 2015). For each of the 220 samples, a FE analysis using the numerical model presented in the previous section is performed. Then, the hydrodynamic pressure distribution when a wave impacts the tank and the horizontal force is maximum is extracted around the AST circumference $(\theta)$ and along the AST height (h). An example of the pressure distribution is presented in Fig. 4 for $S=5.0 \mathrm{~m}, U=0 \mathrm{~m} / \mathrm{s}, H_{w}=1.8 \mathrm{~m}$, and $T_{w}=6.0 \mathrm{~s}$.

\begin{tabular}{|c|c|c|}
\hline Parameter & Parameter name & Range \\
\hline$S(m)$ & Surge height & $1.0-7.5$ \\
\hline$U(\mathrm{~m} / \mathrm{s})$ & Current velocity & $0.0-0.7$ \\
\hline$H_{w}(\mathrm{~m})$ & Wave height & $0.0-2.0$ \\
\hline$T_{w}(\mathrm{~s})$ & Wave period & $3.0-6.0$ \\
\hline
\end{tabular}
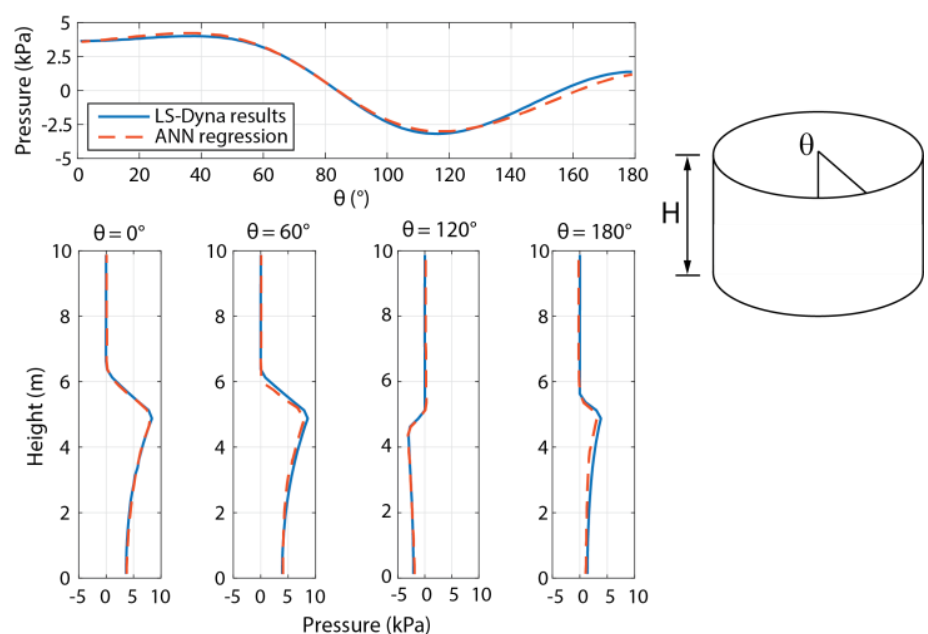

Figure 4. Comparison between the FE model and the ANN regression model for $S=5.0 \mathrm{~m}, U=0 \mathrm{~m} / \mathrm{s} H_{w}=1.8$ $\mathrm{m}$, and $T_{w}=6.0 \mathrm{~s}$. Pressure distribution: a) around the AST circumference; b) along the AST height.

The derived regression model consists of an Artificial Neural Network (ANN) with three hidden layers and 20 neurons per layers. ANNs are employed here given their powerful predictive capabilities; theoretically, ANNs can approximate any continuous function on a bounded domain given an adequate architecture (Murphy 2012). As shown in Eq. 1, the inputs of the derived ANN are the wave parameters (i.e. $S, H_{w}, T_{w}$, and $U$ ) and a point on the tank shell $(\theta, h)$, while the output is the hydrodynamic pressure $\left(P_{d}\right)$ at that same point.

$$
\text { Input: }\left\{S, H_{w}, T_{w}, U, \theta, h\right\} \mapsto \text { Output: }\left\{P_{d}\right\}
$$


On the test samples, the ANN predicted the pressure distributions with a $R^{2}$ value of 0.995 and errors less than $10 \%$ for the maximum pressure. Fig. 4 also shows the adequacy of the ANN model to predict the hydrodynamic pressure distribution for wave conditions never seen before by the model. Given its adequate accuracy and predictive capability, the ANN regression model is used as a surrogate of the FE model in the next sections to facilitate the investigation of the structural behavior of the case study AST and the development of fragility models.

\section{STRUCTURAL BEHAVIOR OF THE AST UNDER SURGE AND WAVE LOADS}

With an adequate estimate of the hydrodynamic loads acting on the case study AST, it is now possible to gain a better understanding of the structural behavior of ASTs during hurricane events. This section focuses specifically on the buckling behavior of the AST under surge and wave loads as this failure mode requires advanced numerical methods for assessment; a predictive model is derived to estimate the buckling strength of the case study AST under surge and wave loads. The vulnerability of ASTs to dislocation can be assessed through simple sums of forces and moments and is detailed directly within the fragility analysis methodology in the next section.

The buckling analyses are performed using LS-Dyna and the FE model shown in Fig. 5. The case study AST is designed according to the American Petroleum Institute (API) 650 standard (API 2013); the AST is designed with a conical roof supported by rafters. The tank shell is modeled using quadrilateral shell elements and an elastoplastic material model with kinematic hardening representing steel. The roof shell is modeled with triangular shell elements while the top angle and roof rafters are modeled with beam elements. The base of the AST is fixed in all directions. The FE model considers both material and geometric nonlinearities. Global imperfections of the tank shell are also included using the method proposed by Kameshwar and Padgett (2015). The internal liquid and the hydrostatic component of the surge are modeled using a triangular pressure distribution. The hydrodynamic loads (i.e. current and waves) are obtained from the previous ANN regression model and applied statically on the tank shell; dynamic effects of the loads are neglected based on the results presented in Bernier and Padgett (2018b) which suggest that they have a negligible influence on the buckling strength.

The critical surge height $\left(S_{c r}\right)$ is the metric employed here to define the buckling strength of the AST. The critical surge height corresponds to the minimum external water level that causes buckling of the tank shell. As explained in Bernier and Padgett (2017), this metric enables an efficient exploration of the effects of each load individually and concurrently on the buckling response of the AST. For a given buckling analysis, the current velocity $(U)$ and the wave parameters $\left(H_{w}, T_{w}\right)$ are fixed, while the external surge height is incremented at each time step using a load control scheme. The hydrodynamic pressure is adjusted accordingly at each time step using the ANN regression model. The surge height when loss of stability occurs corresponds to the critical surge height $\left(S_{c r}\right)$.

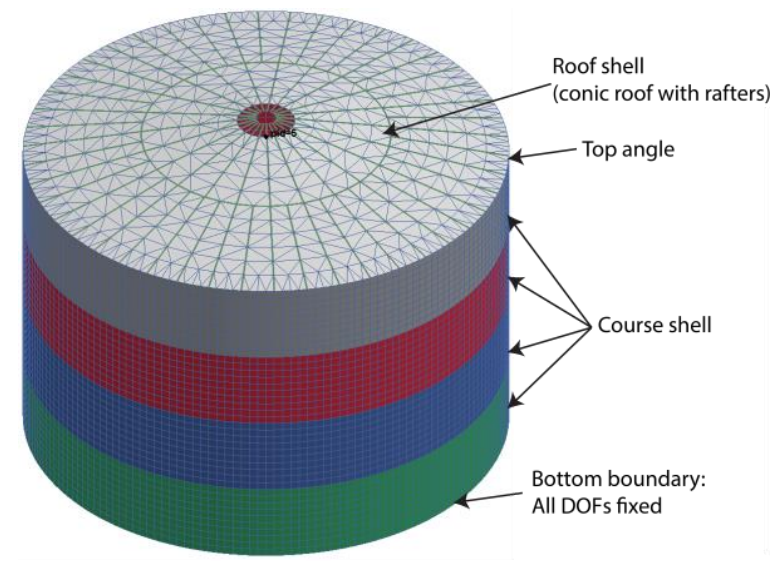

Figure 5. Overview of the finite element model for the buckling analysis.

In order to investigate the effects of the load parameters on the buckling of the case study AST, a predictive model of the buckling strength is derived. A regression model predicting $S_{c r}$ as a function of the AST internal liquid, steel properties, current velocity, and wave parameters is developed using LHS. The surge and wave parameters considered in the LHS design are the same as the ones presented in Table 1, while the AST modeling parameters and their range are summarized in Table 2. The ranges of 
parameters in Table 2 were obtained from Kameshwar and Padgett (2018). A total of 2,000 samples were generated; 1,800 were training samples, while 200 were test samples. This large number of analyses also highlights the benefit of the ANN regression model to estimate hydrodynamic loads; directly using the numerical model in Fig. 1 would have been prohibitive. For each combination of $\rho, L$, $S_{d}, H_{w}, T_{w}$, and $U$, a buckling analysis is performed to determine $S_{c r}$ using the procedure outlined above.

\begin{tabular}{|l|l|l|}
\hline \multicolumn{3}{|c|}{ Table 2. Range of modeling parameters in the LHS design. } \\
\hline Parameter & Parameter name & Range \\
\hline$S_{d}(\mathrm{MPa})$ & Steel design stress & $137-196$ \\
$L(\mathrm{~m})$ & Internal liquid height & $0.0-9.0$ \\
$\rho$ & Relative internal liquid density & $0.5-1.0$ \\
\hline
\end{tabular}

With the $S_{c r}$ values for each of the 1,800 training samples, the following relation was derived using stepwise regression to estimate $S_{c r}$ as a function of the tank parameters and load parameters:

$$
\begin{aligned}
S_{c r} & =6.12-2.02 \rho-7.92 L-2.69 \times 10^{-5} S_{d}+0.29 H_{w}-0.56 T_{w}-0.76 U \\
& +12.76 \rho L-0.13 H_{w} T_{w}+0.91 \rho^{2}+13.13 L^{2}+0.06 T_{w}^{2}-5.53 \rho^{2} L-6.84 L^{3}
\end{aligned}
$$

On the test data, the $R^{2}$ value of this relation is 0.961 and the median error is $3.1 \%$. This model was developed without imposing correlation between hazard parameters. However, surge height, current, and wave parameters are expected to be correlated during a storm. This correlation is a function of other parameters that are site-specific (i.e., fetch and bathymetry). Therefore the approach taken here is to generate a general model that efficiently covers all the combinations of load conditions that could be reasonably encountered at any location during a storm, offering flexibility for broad application across regions that have varying joint hazard intensity probabilities. Hazard correlations can thus be considered when integrating the models herein with site specific hazard scenarios or joint probability density functions of hazard intensity.

Fig. 6 illustrates how varying one parameter at a time, from their lower to upper level (Tables 1 and 2 ), affects $S_{c r}$; when varying a given parameter, all other parameters are kept at their median value. Results show that the internal liquid height has the strongest influence on $S_{c r}$. Product design stress $\left(S_{d}\right)$ has a very limited influence on $S_{c r}$ as buckling occurs in the elastic domain. Current velocity and wave parameters have a significant influence on the buckling response of the AST. Additional analyses also highlighted a significant interaction between the wave height and period. For low periods, increasing the wave height has a limited influence on $S_{c r}$; however, for high periods, it can reduce $S_{c r}$ by $20-25 \%$. Overall, these results indicate that the hydrodynamic component of storm surge as well as wave loads are significant and should be considered when assessing the buckling behavior of ASTs, contrary to existing studies that only considered hydrostatic loads.

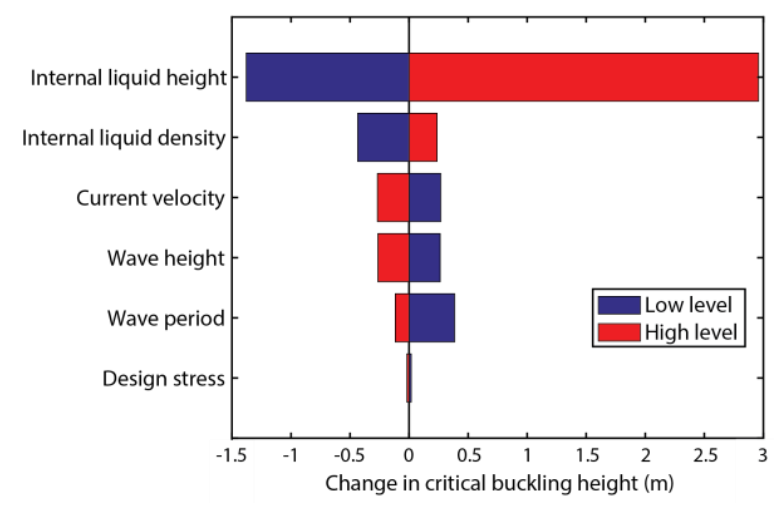

Figure 6. Effects of varying each parameter on the critical surge height.

\section{FRAGILITY ASSESSMENT OF THE AST UNDER SURGE AND WAVE LOADS}

With a good understanding of the hydrodynamic loads and buckling behavior of the case study AST, it is now possible to develop parametrized fragility models for this AST. Parametrized fragility models provide the probability of failure of a structure as a function of the hazard level and the 
structural characteristics. They are useful tools to consider sources of uncertainty when evaluating the structural vulnerability of an AST and can be readily applied for any AST parameters (i.e. internal liquid properties) or surge and wave conditions. As detailed below, parametrized fragility models are developed by evaluating limit state functions with a statistical sampling method. Two fragility models are derived here, one for each of the two failure modes that could occur during storm surge events dislocation from the ground and buckling of the tank shell.

The first step to develop fragility models consists of mathematically representing the failure modes using limit state functions. When considering hydrodynamic loads, three different mechanisms can cause the dislocation of an AST: uplift, overturning, and sliding. The limit state functions for each potential mechanism are presented in Eqs. 3 for uplift, 4 for overturning, and 5 for sliding. Dislocation occurs if any of these three limit state functions is below 0 .

$$
\begin{gathered}
g_{\text {uplift }}=W_{t}+W_{L}-F_{b} \\
g_{\text {overturn }}=\left(W_{t}+W_{L}-F_{b}\right) \cdot D / 2-M_{h d} \\
g_{\text {sliding }}=\left(W_{t}+W_{L}-F_{b}\right) \cdot \varphi-F_{h d}
\end{gathered}
$$

In these equations, $W_{t}$ is the AST self-weight obtained per the API 650 standard; $W_{L}$ is the internal liquid weight and is equal to $\pi g \rho L D^{2} / 4 ; F_{b}$ is the buoyant force from the flood and is equal to $\pi \cdot 10^{3} \cdot g S D^{2} / 4 ; F_{h d}$ and $M_{h d}$ are the horizontal force and overturning moment from the hydrodynamic loads and can be obtained via the ANN regression model; and $\varphi$ is the friction coefficient between the tank foundation and the bottom of the AST. As shown in Fig. 7, these three equations were derived through sums of forces and moments. They indicate that uplift occurs if the buoyant force is larger than the weight of the tank and the stored liquid, overturning occurs if the overturning moment from the hydrodynamic force is larger than the stabilizing moment from the AST and liquid weight, and sliding occurs if the horizontal hydrodynamic force is larger than the sliding resistance provided by friction between the foundation and the bottom AST plate.

In the case of buckling, the limit state function can be expressed as:

$$
g_{\text {buckling }}=S_{c r}-S
$$

In other words, buckling occurs if the external surge height acting on the AST is higher than the critical surge height. As detailed in the previous section, the critical surge height is also function of the internal liquid properties, the current velocity, and the wave parameters.

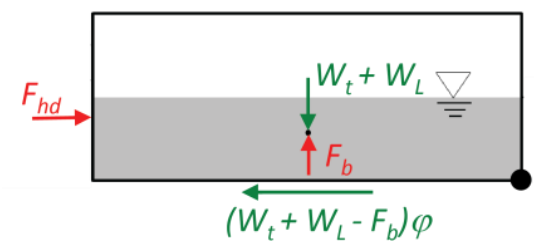

Figure 7. Overview of the forces acting on the AST for dislocation.

The fragility models are then derived using LHS to generate 10,000 training samples and 1,000 test samples. The considered parameters and their range are the same as in Tables 1 and 2 in addition to the friction coefficient; $\varphi$ varies between 0.3 and 0.7 (AFWL 1965, Rabbat and Russell 1985). For each sample (or combination of $\rho, L, \varphi, S, S_{d}, H_{w}, T_{w}$, and $U$ ), $F_{h d}$ and $M_{h d}$ are obtained based on the estimate of hydro dynamic pressure from the ANN regression model presented above, while $S_{c r}$ is estimated from Eq. 2. The limit state functions are then evaluated to determine if the sample buckled or floated. With the training samples, logistic regression is then employed to obtain the fragility models, one for dislocation and one for buckling. As shown in Eq. 7, the fragility models are conditioned on $\rho, L, S, H_{w}$, $T_{w}, U$, and $\varphi$.

$$
P\left(\text { Failure } \rho, L, \varphi, S, H_{w}, T_{w}, U\right)=\frac{1}{1+\exp \left(-l\left(\rho, L, \varphi, S, H_{w}, T_{w}, U\right)\right)}
$$


In this equation, $l(\cdot)$ is a logit function given by Eqs. 8 for dislocation and 9 for buckling. Only the most influential parameters and polynomial combinations of parameters were considered in the logit functions by using stepwise regression. As the steel design stress $\left(S_{d}\right)$ has a limited effect on both buckling and dislocation, it was not retained as a parameter of the logit functions.

$$
\begin{aligned}
l\left(\rho, L, \varphi, S, H_{w}, T_{w}, U\right)= & -5.6+1.2 \rho+1.7 L+13.4 S+2.7 H_{w}-0.3 T_{w} \\
& -1.2 U+7.3 \varphi-13.4 \rho L+0.6 L \cdot U-4.3 L \varphi \\
& +2.4 S \cdot H_{w}+0.1 S \cdot T_{w}-0.4 H_{w} T_{w}+0.8 S \cdot H_{w} T_{w} \\
l\left(\rho, L, S, H_{w}, T_{w}, U\right)= & -29.8+5.7 \rho+3.9 L+7.1 S-3.2 H_{w}-0.4 T_{w} \\
& +4.5 U-3.4 \rho L+0+1.2 H_{w} T_{w}-0.9 L^{2}+0.1 L^{3}
\end{aligned}
$$

Finally, the accuracy of the fragility models is verified using the test samples. On the test data, the accuracy of the logistic regression classifiers is $99.5 \%$ and $97.5 \%$ for dislocation and buckling respectively. The above fragility models are derived by considering all load parameters uncorrelated. As explained previously, this allows more general models to estimate the probability of failure of the AST under any load conditions that could be reasonably observed during a storm. The correlation between the surge and wave loads should be included in the posterior analysis (i.e. risk assessment). Using Eq. 7 requires knowledge of all the conditioning parameters. However, if some parameters are unknown or uncertain, it is also possible to condition the fragility models on a smaller subset of parameters by convolving Eq. 7 over the probability distributions of the uncertain parameters via MCS or numerical integration. This approach was used to obtain the curves shown in Figs. 8 and 9, where the fragility models are only conditioned on $S ; \rho, H_{w}, T_{w}, U$, and $\varphi$ are fixed, while $L$ is uniformly distributed with lower and upper bounds of 0 and $9 \mathrm{~m}$.

The fragility models provide key insights regarding the effect of wave and surge parameters on the probability of failure of the case study AST. Fig. 8 shows the effects of surge height, wave height, and wave period on the dislocation fragility, while Fig. 9 shows the same analysis for the buckling fragility; in these figures, $\rho=0.75, U=0.35$, and $\varphi=0.5$. Fig. 8 indicates that the case study AST becomes vulnerable to dislocation for surge levels as low as $0.25 \mathrm{~m}$; the buoyant force of $0.25 \mathrm{~m}$ of water corresponds approximately to the self-weight of the AST if it is empty. After this point, the probability of dislocation increases linearly and reaches a value of 1.0 for surge height between 5 and $7 \mathrm{~m}$ depending on the presence of waves or not. In the case of buckling, the AST becomes vulnerable for surge height above $3 \mathrm{~m}$. The probability of buckling then increases in a nonlinear way and reaches values above 0.8 for $7 \mathrm{~m}$ of surge. Figs 8 and 9 also show that the probability of dislocation is always larger than the probability of buckling. This indicates that buckling might not be a critical failure mode for unanchored ASTs of similar dimensions as the case study and highlights the need to explore a range of tank geometries as well as develop fragility models for anchored ASTs in future research; buckling might be the dominant failure mode if the AST is restrained to the ground. At last, results in Figs. 8 and 9 also indicate that for low wave periods the influence of wave loads is negligible. However, for longer wave periods, wave effects are significant, and neglecting them could underestimate the probability of failure by up to $20 \%$ and $30 \%$ for dislocation and buckling respectively. Also, the effects of waves are primarily significant for high surge levels for dislocation, while they are significant for low surge levels in the case of buckling. In the case of current, the effects of current on fragility are minimal for dislocation while they are significant for buckling; current can affect the buckling fragility by up to $10 \%$. Again, these results highlight the importance of considering the hydrodynamic effect of the surge as well as wave loads when evaluating the structural vulnerability of an AST. 

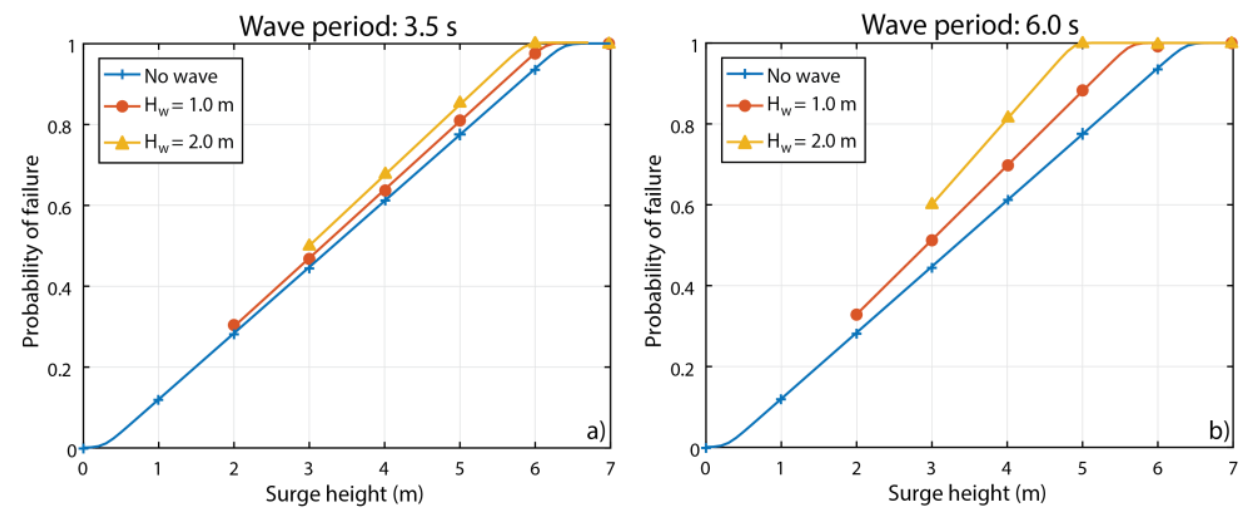

Figure 8. Dislocation fragility curves for the case study AST. a) Wave period of $3.5 \mathrm{~s}$; b) Wave period of $6.0 \mathrm{~s}$.
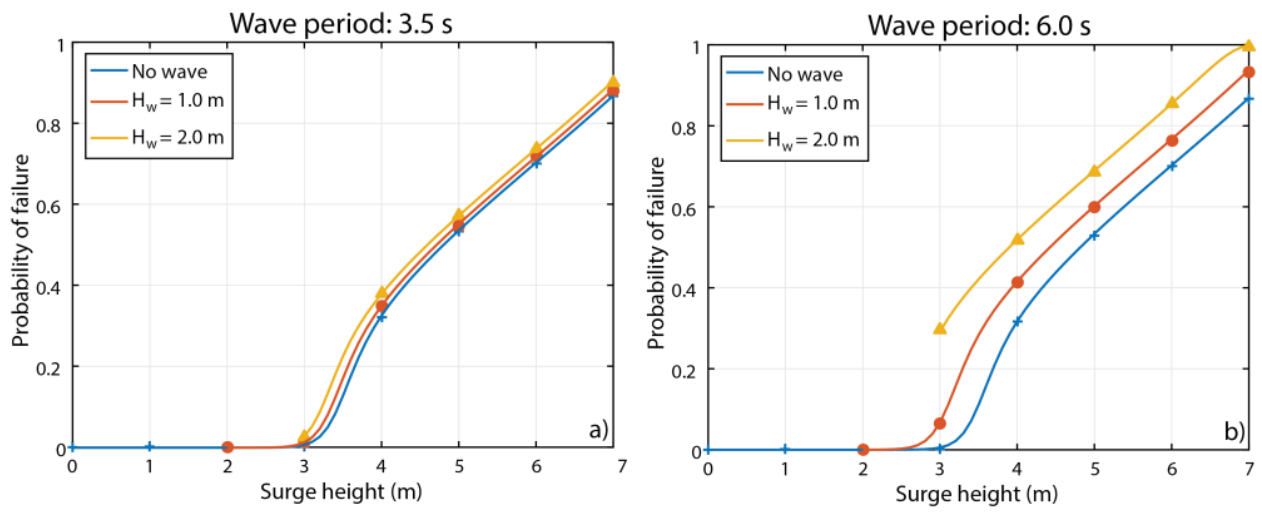

Figure 9. Buckling fragility curves for the case study AST. a) Wave period of $3.5 \mathrm{~s}$; b) Wave period of $6.0 \mathrm{~s}$.

\section{CONCLUSIONS}

This study presented a methodology to explore the structural behavior and develop fragility models for an AST subjected to hydrodynamic effects of storm surge and wave loads. First, a finite-element model that takes into account FSI was developed to compute hydrodynamic loads acting on a case study AST during storm surge. Validation of this model against experimental results also indicated that the modeling assumptions were adequate to determine hydrodynamic loads on the AST. While this FE model provided accurate estimates of loads acting on the AST, its high computational cost was a major drawback for the development of fragility models. Thus, a surrogate model, based on Artificial Neural Network and a small set of FE analyses, was also developed to facilitate the estimation of hydrodynamic loads across ranges of surge and wave conditions. Next, the structural behavior of the case study AST under surge and wave loads was investigated. Using the hydrodynamic loads predicted by the surrogate model, a series of buckling analyses were performed to investigate the effects of modeling parameters and load conditions on the buckling behavior of the case study AST. The buckling analyses were performed using a nonlinear FE model of the storage tank alone. Results of the buckling analyses were also used to derive a regression model predicting the buckling strength as a function of the AST internal liquid, steel properties, and load conditions. The regression model indicated that the AST internal liquid properties had the strongest effect on the buckling strength of the case study tank. Results also indicated that current and wave loads could affect the buckling strength by up to $25 \%$ and that such loads should be considered when assessing the buckling behavior of ASTs. Lastly, parametrized fragility models were developed to assess the probability of dislocation or buckling of the case study AST. These fragility models can now be used to readily assess the structural vulnerability of the AST under a range of reasonable surge and wave conditions. These models also provided useful insights regarding the effects of load conditions on the probability of failure of the AST. Again, results highlighted the importance of considering hydrodynamic loads, contrary to existing studies which usually neglect them. Neglecting wave loads can underestimate the probability of failure of the case study AST by up to $30 \%$ especially when long wave periods are expected, while neglecting current 
effects can underestimate the probability of failure by up to $10 \%$. Even though the structural and fragility analyses presented here were performed for a single AST, the methodology proposed in this study opens the path for future work to assess the vulnerability of ASTs across a range of geometries and design details. Thus, future work will develop fragility models also parametrized on the geometry as well as fragility models for anchored ASTs.

\section{ACKNOWLEDGMENTS}

The authors acknowledge the financial support of the National Science Foundation under award \#1635784. The contributions of the first author were also supported in part by the Natural Sciences and Engineering Research Council of Canada. The authors also thank Prof. Clint Dawson for providing the $A D C I R C+S W A N$ results. Any opinions, findings, and conclusions or recommendations expressed in this paper are those of the authors and do not necessarily reflect the views of the sponsors.

\section{REFERENCES}

Air Force Weapons Laboratory (AFWL). 1965. Experimental Study of Static and Dynamic Friction between Soil and Typical Construction Materials, Technical Report AFWL-TR-65-161, Kirtland Air Force Base, NM.

American Petroleum Institute (API). 2013. Standard 650: Welded Steel Tanks for Oil Storage, Washington, DC.

Bernier, C., and J.E. Padgett. 2017. Effects of combined surge, wave, and wind loads on the buckling of aboveground storage tanks, Proceedings of 13th Americas Conf. on Wind Engineering, AAWE.

Bernier, C., and J.E. Padgett. 2018a. Forensic investigation of aboveground storage tank failures during Hurricane Harvey using fragility models, Proceedings of Forensic Engineering 8th Congress, ASCE.

Bernier, C., and J.E. Padgett 2018b. Dynamic buckling of aboveground storage tanks subjected to hurricane-induced waves, Proceedings of Structure Congress 2018, ASCE, 530-540.

Bernier, C., Y. Lin, J.E. Padgett, C. Dawson, P. Lomonaco, D. Cox. 2017. Large-scale laboratory experiments of wave impacts on vertical cylinders. DesignSafe-CI [publisher], Experiments dataset, doi:10.17603/DS27D4G.

Cozzani, V., M. Campedel, E. Renni, and E. Krausmann. 2010. Industrial accidents triggered by flood events: Analysis of past accidents, J. Hazard. Mater., 175(1), 501-509.

Dawson C.N. 2017. The Computational Hydraulics Group, http://chg.ices.utexas.edu/.

Fenton, J.D. 1988. The numerical solution of steady water wave problems, Computers and Geosciences, 14(3), 357-368.

Fenton, J.D. 2015. Use of the programs FOURIER, CNOIDAL and STOKES for steady waves, John D. Fenton, Austria.

Godoy, L.A. 2007. Performance of storage tanks in oil facilities damaged by Hurricanes Katrina and Rita, J. Perform. Constr. Facil., 21(6), 441-449.

Godoy, L.A. 2016. Buckling of vertical oil storage steel tanks: Review of static buckling studies, ThinWalled Struct., 103, 1-21.

Hyder, M. 2008. Oil Spill Intelligence Report, Assessment of Hurricane Ike Damage Continues, Aspen Publishers.

Kameshwar, S., and J.E. Padgett. 2015. Stochastic modeling of geometric imperfections in above ground storage tanks for probabilistic buckling capacity estimation, ASCE-ASME J. Risk Uncertainty Eng. Syst. Part A Civ. Eng., 2(2), C4015005.

Kameshwar, S., and J.E. Padgett. 2018. Storm surge fragility assessment of above ground storage tanks, Structural Safety, 70, 48-58.

Kriebel, D.L. 1990. Nonlinear wave interaction with a vertical circular cylinder. Part I: Diffraction theory, Ocean Enginering, 17, 345-377.

Landucci, G., G. Antonioni, A. Tugnoli, and V.Cozzani. 2012. Release of hazardous substances in flood events: Damage model for atmospheric storage tanks, Reliab. Eng. Syst. Saf., 106, 200-216.

Lawrence Livermore Technology Corporation (LSTC) (2015). LS-Dyna R8.0 [Computer software], Livermore, CA.

McKay, M.D., R.J. Beckman, and W.J. Conover. 1979. Comparison of three methods for selecting values of input variables in the analysis of output from a computer code, Technometrics, 21(2), 239-245. 
Morison, J.R., J.W. Johnson, and S.A. Schaaf. 1950. The Force Exerted by Surface Waves on Piles, J Pet Technol, 2, 149-154.

Murphy, K.P. 2012. Machine Learning: A Probabilistic Perspective, MIT Press, Cambridge.

Myers, P. 1997. Aboveground Storage Tanks, McGraw-Hill, New York, 690 pp.

Palinkas, L., M. Downs, J. Petterson, and J. Russell. 1993. Social, cultural, and psychological impacts of the Exxon Valdez oil spill, Hum. Organ., 52(1), 1-13.

Pardue, J.H. 2013. Severe storms and bulk chemical storage, Proceedings Hurricane Ike: 5 Years Later Conf., Rice University.

Rabbat, B.G., and H.G. Russel. 1985. Friction Coefficient of Steel on Concrete or Grout, J. Struct. Eng., 111(3), 505-515.

Sakakiyama, T., S. Matsuura, and M. Matsuyama. 2009. Tsunami force acting on oil tanks and buckling analysis for tsunami pressure, J. Disaster Res., 4(6), 427-434.

Santella, N., L.J. Steinberg, and H. Sengul. 2010. Petroleum and hazardous material releases from industrial facilities associated with Hurricane Katrina, Risk analysis, 30(4), 635-649. 